See discussions, stats, and author profiles for this publication at: https://www.researchgate.net/publication/291234104

\title{
A New Method for Sclera Vessel Recognition Using OLBP
}

Chapter · January 2013

DOI: 10.1007/978-3-319-02961-0_46

\section{CITATIONS}

16

4 authors, including:

Abhijit Das

University of Southern California

42 PUBLICATIONS 296 CITATIONS

SEE PROFILE

Miguel A. Ferrer

Universidad de Las Palmas de Gran Canaria

281 PUBLICATIONS 2,943 CITATIONS

SEE PROFILE

Some of the authors of this publication are also working on these related projects:

STEFANN View project

Project Handwritten document image segmentation View project

\section{READS}

40

Umapada Pa

Indian Statistical Institute

396 PUBLICATIONS 7,117 CITATIONS

SEE PROFILE 


\title{
A New Method for Sclera Vessel Recognition using OLBP
}

\author{
Abhijit Das $^{1 *}$, Umapada Pal $^{2}$, Miguel A. Ferrer Ballester ${ }^{3}$, Michael Blumenstein ${ }^{1}$, \\ ${ }^{1}$ Institute for Integrated and Intelligent Systems, Griffith University, Queensland, Australia, \\ ${ }^{2}$ Computer Vision and Pattern Recognition Unit, Indian Statistical Institute, Kolkata, India, \\ ${ }^{3}$ IDeTIC, University of Las Palmas de Gran Canaria, Las Palmas, Spain, \\ abhijit.das@griffithuni.edu.au,umapada@isical.ac.in, mferrer@dsc.ulpgc.es, \\ m.blumenstein@griffith.edu.au
}

\begin{abstract}
This paper proposes a new sclera vessel recognition technique. The vessel patterns of sclera are unique for each individual and this can be utilized to identify a person uniquely. In this research we have used a time adaptive active contour-based region growing technique for sclera segmentation. Prior to that, we have made some tonal and illumination correction to get a clearer sclera area without the distributing vessel structure. This is because the presence of complex vessel structures occasionally affects the region-growing process. The sclera vessels are not prominent in the images, so in order to make them clearly visible, a local image enhancement process using a Haar high pass filter is incorporated. To get the total orientation of the vessels, we have used Orientated Local Binary Pattern (OLBP). The OLBP images of each class are used for template matching for classification by calculating the minimum Hamming Distance. We have used the UBIRIS version 1 dataset for the experimentation of our research. The proposed approach has achieved high recognition accuracy employing the above-mentioned dataset.
\end{abstract}

Keywords: Sclera Biometric, Sclera vessels, Patterns, OLBP, LBP, Haar filter.

\section{Introduction}

Biometrics refers to automatic authentication of individuals based on their physiological and behavioral characteristics. A large number of research works have been performed in the field of biometrics over the last few decades. But no single biometric technique can be applied universally. So, further research on biometric traits is required. Sclera recognition is considered to be a good trait to complement traditional traits, as sclera is a highly-protected portions of the eye. Personal identification by the vessel pattern of the sclera is possible because these patterns possess a high degree of randomness and this makes it ideal for personal identification.

The various steps involves in sclera recognition are accurate segmentation of the sclera area, sclera vessel enhancement and the extraction of discriminative features of the sclera vessel pattern for authentication and identification purposes. Objective also focuses that the authentication system should work in real-time so that

\footnotetext{
* Corresponding author: Abhijit Das, Institute for Integrated and Intelligent Systems, Griffith University, email: abhijit.das@griffithuni.edu.au.
} 
extraction, representation and comparison of texture images should not consume large computational resources.

This paper proposes a whole biometric scheme for personal identification based on sclera trait. We propose a new preprocessing technique for vein highlighting which makes it possible to apply region growing based algorithm for sclera segmentation. Otherwise the vein inside the sclera creates hindrance to standard region growing. The sclera parameterization based on OLBP is also new in the literature. The OLBP texture measure is judged appropriate for sclera segmentation because it is able to improve the vein structure representation by the vein trajectory orientation statistic. The hamming distance is used as sclera identification score here.

The organization of the paper is as follows: Section 2 explains the proposed approach of segmentation, preprocessing of the sclera images, followed by the sclera vessel enhancement process, feature extraction and classification. In Section 3 the experimental details are given, and Section 4 draws the overall conclusions.

\section{Proposed Approach}

Several approaches are proposed on sclera biometric in the literature. To our knowledge, first recognized work on sclera biometric is recorded in [1]. Automatic segmentation processes of sclera are proposed in [4], [6] and many features like LBP [9], GLCM [8] are used for recognition. Pieces of work on multi-angled sclera recognition $[2,7]$ well as multimodal eye recognition techniques $[3,5,10]$ are also proposed using sclera and iris. In this section we explain a new sclera recognition technique. The content of this section includes a sclera segmentation process, a sclera vein enhancement technique, feature extraction of sclera texture patterns and sclera image registration. This is finally followed by the classification technique.

\subsection{Sclera Segmentation}

Generally the portion of blood vessels inside the sclera region is randomly-oriented which creates a pattern. As they are oriented in different layers, the intensity of the vessels varies highly. Some of them are quite bright and bundled. And this creates a hindrance to standard region growing. So we have considered the red channel of the image for segmentation, as the blood vessels are less prominent here as shown in figure 1(b). A representation for each color channel is indicated in Figure 1.

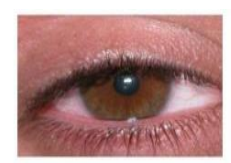

$1(\mathrm{a})$

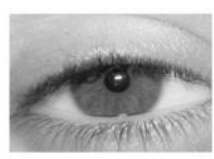

$1(b)$

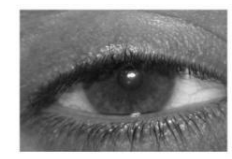

$1(\mathrm{c})$

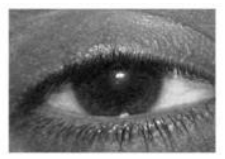

$1(d)$

Fig. 1. (a) The image of the original RGB image, (b) The red channel component of 1(a), (c) The Green channel component of 1(a), and 1(d) blue channel component of 1(a), 
Adaptive histogram equalization is performed with a small window of $2 \times 2$ to reduce the vessel content. Next to which we use a bank of low pass Haar reconstruction filters to get a clear white sclera without the vessel. The filter is used with a high cut off. Analyzing different results, the cut off value that produces the best result is determined and used for experimentation. The figure 2(a) is the histogram equalized image and 2(b) the Haar filtered image of 2(a).

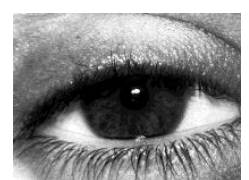

2(a)

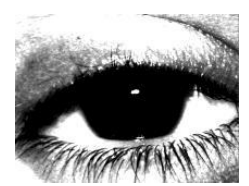

2(b)

Fig. 2. (a) is the histogram equalized image and (b) the Haar filtered image of 2(a).

This preprocessed image can be used for segmentation by a time adaptive active contour-based region growing segmentation method in [11]. The right and the left sclera are segmented separately. For region growing-based segmentation, we need a seed point. In order to get the seed point we use Daugmans integro-differential method [12], which is used to calculate the center of the iris. From the center of the iris at a distance of 1.1 of the radius length of the iris and a deviation of 45 degrees with the horizontal, the seed point for region growing is set in both sides of the sclera as explained in Figure 3.

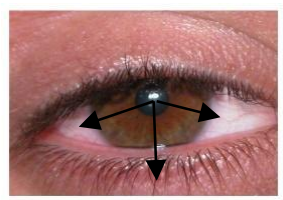

Fig. 3. Seed point for sclera segmentation

Now the seed point grows to provide the total sclera region as explained in Figure 4.

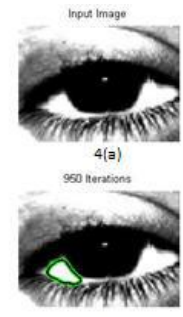

$4(c)$

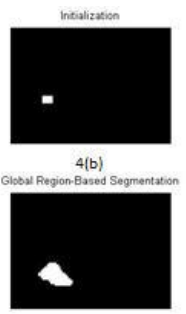

4(d)

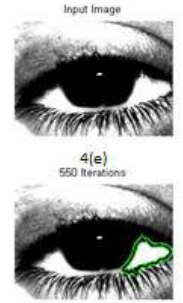

$4(\mathrm{~g})$

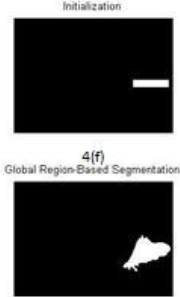

$4(\mathrm{~h})$

Fig. 4. Region growing segmentation method of left and right sclera. 4(a) The Histogram equalized and filtered image of red component of 1(a),4(b) Initial size of the seed for right sclera,4(c) Segmented image of 4(a),4(d) Segmented mask developed for right sclera, 4(e)The 
Histogram equalized and filtered image of red component of 1(a),4(f) Initial size of the seed for left sclera,4(g) Segmented image of 4(e), 4(h) Segmented mask developed for left sclera

The green channel images of the RGB are masked by the segmented mask to get the region of interest as shown in Figure 5. The green channel image is used because here the vessel patterns look most prominent as shown in Figure 1(c).

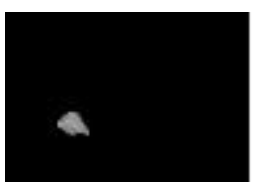

$5(a)$

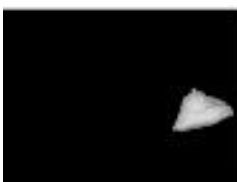

$5(\mathrm{~b})$

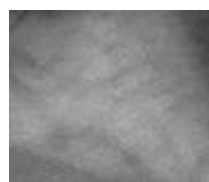

$5(a)$

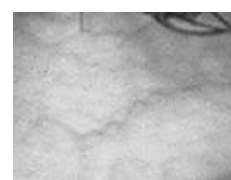

$5(\mathrm{~b})$

Fig. 5. (a) \& (b) Segmented region of interest. (c) \& (d) a microscopic view of the ROI

\subsection{Sclera vein structure enhancement}

The vessels in the sclera are not prominent, so in order to make them clearly visible, image enhancement is required. Adaptive histogram equalization is performed with a large window size of $42 \times 42$ to make the vessel structure more prominent as shown in Figure 6.

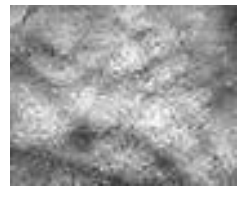

6(a)

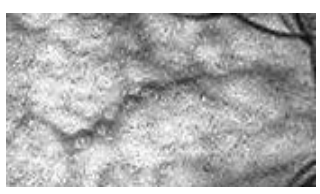

$6(b)$

Fig. 6. The adaptive histogram images of the vessels in 5(c) \& 5(d).

Then a bank of high pass decomposition Haar wavelet multi-resolution filters is used for obtaining the final enhanced vessel structure. The filter is used with a high cut off. The cut off value is determined empirically; the cut off value that produced the best result was used for experimentation. A Median filter is used to reduce some noise that is present in the surrounding area of the vessel. The images of the enhanced vessels following Haar filtering are provided in Figure 7.

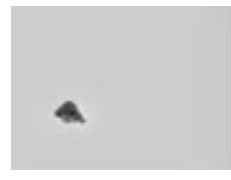

$7(\mathrm{a})$

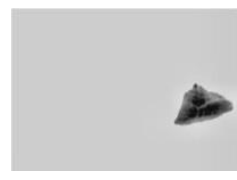

7(b)

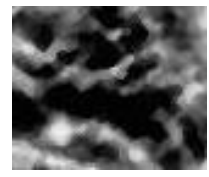

7(a)

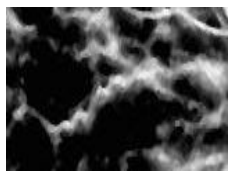

7(b)

Fig. 7. 7(a) \& 7(b) The images of enhanced vessel 7(c) \& 7(d), a microscopic view of 7(a) \& $7(b)$. 


\subsection{Feature extraction method}

Local patterns, such as LBP (Local Binary Patterns), can be seen as a unifying approach to the traditionally statistical and structural approaches of texture analysis. Applied to black and white images, an LBP can be considered as the concatenation of the binary gradient directions. This contains micro-pattern information of the distribution of the edges, spots, and other local figures in an image which can be used as features for sclera recognition. Local patterns used for sclera features and the classifier used for sclera identification are discussed as follows. The original LBP operator labels the pixel of an image by thresholding the $3 \times 3$ neighbourhood of each pixel and concatenating the results binomially to form a number. Assume that a given image is defined as $I(Z)=I(x, y)$. The LBP operator transforms the input image to $(Z)$ as follows:

$$
\operatorname{LBP}\left(Z_{c}\right)=\sum_{p=0}^{7} s\left(I\left(Z_{p}\right)-I\left(Z_{c}\right)\right) \cdot 2^{p},
$$

Where $s(l)=\left\{\begin{array}{ll}1 & l \geq 0 \\ 0 & l<0\end{array}\right.$ is the unit step function and $I\left(Z_{p}\right)$ is the 8-neighborhood around $I\left(Z_{c}\right)$. The feature representation method called Orientated Local Binary Pattern (OLBP) [13] is an extension of the local binary pattern (LBP). OLBP can represent more explicitly the orientation information of the strokes which is an important characteristic of scripts. The $O L B P$ of a given pixel $Z_{c}$ is computed as follows:

1. Compute the sequence $s\left(I\left(Z_{p}\right)-I\left(Z_{c}\right)\right), p=0, \ldots, 7$.

2. Find the starting index (Start) and ending index (End) of the longest continuous 0 substring looking cyclically in the sequence of the previous step. $\quad$ Start $=\operatorname{argmax}(($ StringLength $)), \quad$ End $=$ Start + StringLength $($ StartOri $)-1$,

3. The index of the zeros substring center is the $O L B P$, i.e.

$$
\operatorname{OLBP}\left(Z_{c}\right)=\operatorname{round}((\operatorname{Star} t+E n d) / 2) \bmod 8 \text {, }
$$

Where round() rounds a number to the nearest integer, and mod is the arithmetic complement operation. An example of OLBP images of the vessel structure are presented in Figure 8.
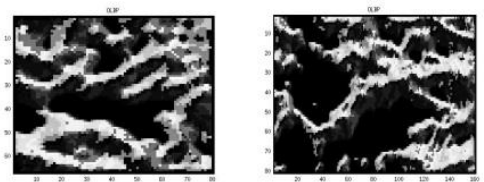

Fig. 8. OLBP of the vein images

\subsection{Image Registration}

In order to make image translation independent, we have registered the iris centre to the centre of the image for each of the OLBP images produced in the earlier section. Registration is performed by using the following equations. 


$$
\begin{aligned}
\text { rowdiff } & =\operatorname{Imgx}-\mathrm{Ix} \\
\text { columndiff } & =\mathrm{Imgy}-\mathrm{Iy}
\end{aligned}
$$

where $(I x, I y)$ represents the iris centre location, $(\operatorname{Imgx}, \operatorname{Imgy})$ is the image centre, and (rowdiff, columndiff) denotes the relative distance of the iris centre from the image centre. Now the relative amount of shift in row direction and column direction is applied to the OLBP image to register it against the image centre.

\subsection{Classification}

Template-based matching is used for classification. The gallery of query images is overlapped over the template of each class. The OLBP regions are binarized to get a binary template. Subsequently, identification is performed by template matching over the OLBP region as the Hamming Distance between the query image and templates, the matching which creates the minimum Hamming Distance is considered as the class of the query image. The Hamming Distance is calculated as below.

$$
\mathrm{HD}=\sum \frac{(\text { template } \oplus \text { query }) \cap(\text { mask } \cup \text { query mask })}{\text { template }-\sim(\text { mask } \cup \text { query mask })}
$$

Mask and query mask are the masks of the template and query image. The symbol $\bigoplus$ signifies the XOR operation, the symbol $\cap$ signifies the AND operation, the symbol U signifies the OR operation, - signifies subtraction operator and the symbol $\sim$ signifies the NOT operation.

\section{Experiments and results}

The experimental setup and the results of our proposed work are explained in this section.

\subsection{Data Set}

In order to evaluate the performance of our proposed method, the UBIRIS v1 database [14] is utilized for our experiments. This database consists of 1877 RGB images taken in two distinct sessions (1205 images in session 1 and 672 images in session 2) from 241 identities where each channel of RGB color space is represented in grey-scale. The database contains blurred images and images with blinking eyes. Both high resolution images $(800 \times 600)$ and low resolution images $(200 \times 150)$ are provided in the database. All the images are in JPEG format. We have used different quality of images. Some of them are not occluded having good quality of sclera regions visible, some of them are of medium quality and the third type is of poor quality with respect to sclera region visibility.

For our experimentation we have considered the images of session 1. One image from the session is randomly chosen and utilized for template generation and the other four were used as query images. So we have $241 * 4$ scores for FRR and $242 * 241$ score for FAR statistics. Among this 54 images were discarded because of failures 
during acquire. All the stimulation experiments performed here were developed in Matlab

\subsection{Experimental Results}

The experiments have been conducted to work out the sclera identification capability of the proposed features and classifier. As it is a classical identification or verification problem, the results will be given in terms of Cumulative Matching Curves (CMC) and Equal Error Rate (EER) curve. The CMC \& EER curve is displayed in Figure 9. Along the $\mathrm{X}$ - axis we have the rank of the CMC and along the $\mathrm{Y}$ - axis the identification rate. Based on the interpretation of the graph it can be noted that faithful accuracy is achieved. Along the $\mathrm{X}$ - axis we have the matching score and along the $\mathrm{Y}$ axis the density.
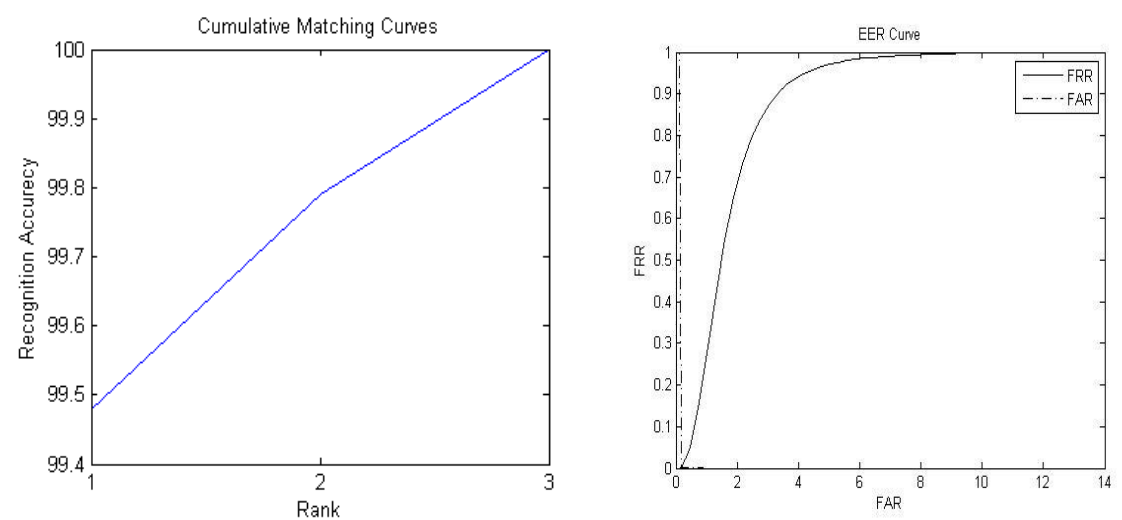

Fig. 9. CMC curve for recognition \& EER curve for recognition

Table 1 provides the numerical data of Equal Error Rate and the Cumulative Matching Curves of the verification experiment.

Table 1. Equal Error Rate and the Cumulative Matching of the verification

\begin{tabular}{ccc}
\hline Dataset & EER(\%) & Cumulative Matching \\
\hline UBIRIS version 1 & 0.52 & $99.48 \%$ \\
\hline
\end{tabular}

The results of the proposed work is put in perspective with the state-of-the-art by analyzing it with the most similar work on UBIRIS version 1, we could find in the literature. Table 2 reflects a state-of-the-art analysis of the most similar work on UBIRIS version 1.

Table 2. The state-of-the-art analysis the most similar work on UBIRIS version 1

\begin{tabular}{cc}
\hline Work & Equal Error Rate ( in \%) \\
\hline Zhou et al. [4] & 1.34 \\
Oh et al. [9] & 0.47 (manual segmentation for some images ) \\
Proposed System & 0.52 \\
\hline
\end{tabular}




\section{Conclusions}

This paper deals with a novel method of sclera recognition. We have proposed a sclera preprocessing algorithm to whiten the sclera eye portion, which improves the accuracy of the active contour procedure proposed for sclera segmentation. The vessel pattern has been enhanced with adaptive histogram equalization and a high pass Haar filter for establishing appropriate features. The OLBP provides information about the different pattern structures of sclera. Identification is accomplished by template matching over the OLBP region as minimum hamming distance matching. The proposed approach has achieved high recognition accuracy employing the UBRIS version1 dataset.

\section{References}

1. Derakhshani, R., Ross, A., Crihalmeanu, S.: A new biometric modality based on conjunctival vasculature. Proc. of Artificial Neural Networks in Engineering, pp. 1-8 (2006).

2. Zhou, Z., Du, Y., Thomas, N. L., Delp E. J.: Multi-angled sclera recognition. IEEE Workshop on Computational Intelligence in Biometrics and Identity Management, pp. 103 -108 (2011).

3. Zhou, Z., Du, Y., Thomas, N. L., Delp E. J.: Multimodal eye recognition. Proceedings of the International Society for Optical Engineering, vol. 7708(770806), pp.1-10 (2010).

4. Zhou, Z., Du, Y., Thomas, N. L., Delp E. J.: A new biometric sclera recognition. IEEE transaction on System, Man And Cybernatics -PART A: System And Human, vol. 42(3), pp. 571-583 (2012).

5. Zhou, Z., Du, Y., Thomas, N. L., Delp E. J.: Quality Fusion Based Multimodal Eye Recognition, IEEE International Conference on Systems, Man, and Cybernetics, pp. 12971302 (2012).

6. Khosravi, M. H., Safabakhsh, R.: Human eye sclera detection and tracking using a modified time-adaptive self-organizing map. Pattern Recognition, vol. 41, pp. 2571 - 2593 (2008).

7. Crihalmeanu, S., Ross, A.: Multispectral sclera patterns for ocular biometric recognition. Pattern Recognition Letters, vol. 33, pp. 1860-1869 (2012).

8. Tankasala, S. P., Doynov, P., Derakhshani, R. R., Ross, A., Crihalmeanu, S.: Biometric Recognition of Conjunctival Vasculature using GLCM Features. International Conference on Image Information Processing, pp. 1-6 (2011).

9. Oh, K., Toh, K.: Extracting Sclera Features for Cancelable Identity Verification, 5th IAPR International Conference on Biometric: 245-250, (2012).

10. Gottemukkula, V., Saripalle, S. K., Tankasala, S. P., Derakhshani, R., Pasula, R., Ross, A.: Fusing Iris and Conjunctival Vasculature: Ocular Biometrics in the Visible Spectrum. IEEE Conference on Technologies for Homeland Security, pp. 150-155 (2012).

11. Chan, T. F., Luminita, A. V.: Active Contours Without Edges. IEEE Transaction On Image Proccessing, vol. 10( 2), pp. 266-277( 2001)

12. Daugman, J. G.: High confidence visual recognition of persons by a test of statistical independence. IEEE Transactions on Pattern Analysis and Machine Intelligence, vol. 15(11), pp.1148-1161 (1993).

13. Bu, W., Wub, X., Gaob, E.: Hand Vein Recognition Based on Orientation of LBP. Proc. of SPIE, Vol. 8371(83711), pp. 1-12 (2012).

14. UBIRIS version 1 dataset for eye, Available at, (http://iris.di.ubi.pt/). 